\title{
Beschneiungsspeicher und Anlagentechnik
}

In diesem Heft zum Themenschwerpunkt „Beschneiungsspeicher und Anlagentechnik“ wird versucht, einen aktuellen Überblick zu Planungsgrundsätzen, Technologien sowie Bau- und Betriebserfahrungen in Österreich zu geben.

Um den Erwartungen des Tourismus und der Sportbegeisterten zu entsprechen, sind Beschneiungsund Speicheranlagen zur Gewährleistung der Schneesicherheit zu einem unerlässlichen Bestandteil von Wintersportregionen geworden. Abhängig von den zu beschneienden Pistenflächen wird eine ausreichende Wasserbevorratung zur künstlichen Schneeerzeugung in allen Höhenlagen immer dringender und in den meisten Regionen sind in Zeiten des Wasserbedarfs zur Beschneiung zu geringe Wasserressourcen aus den Vorflutgewässern verfügbar. Daher kann nur eine Wasserbevorratung durch ausreichend bemessene Speicherkapazitäten die gestellten Anforderungen erfüllen.

Aus den verfügbaren Statistiken der Österreichischen Seilbahnwirtschaft lässt sich ableiten, dass über $70 \%$ der Winterurlauber wegen des Skisports nach Österreich kommen und dabei jährlich mehr als 50 Millionen Skifahrertage erzielt werden. Die Skigebiete verfügen über etwa 23.000 ha Pistenflächen, wovon ca. $60 \%$ oder etwa 14.000 ha beschneibar sind; dies würde bei einer mittleren Pistenbreite von etwa 40 Metern einer Pistenlänge von insgesamt rund $3.500 \mathrm{~km}$ entsprechen. Wenn davon ausgegangen wird, dass jährlich je Hektar Pistenfläche zwischen etwa $2.500 \mathrm{~m}^{3}$ bis $3.500 \mathrm{~m}^{3}$ bzw. im Mittel ca. $3.000 \mathrm{~m}^{3}$ Wasser für die künstliche Beschneiung benötigt werden, ergibt dies zur Grund- und Nachbeschneiung für alle österreichischen Beschneiungsanlagen einen Bedarf in einer Größenordnung von etwa 42 Millionen $\mathrm{m}^{3}$ Wasser. Dieses Volumen kann nur über eine sorgsame Wasserbewirtschaftung sowie Wassernutzung und mit einer Umlagerung sowie Speicherung aufgebracht werden. Dafür stehen etwa 420 Speicherbecken österreichweit zur Verfügung. Es bedurfte einerseits besonderer Anstrengungen, um die erforderlichen Speicherkapazitäten zu schaffen und geordnet zu betreiben, sowie andererseits eines außergewöhnlichen Investitionswillens und -bedarfs zur Bewältigung der gestellten Aufgaben. Der wasserwirtschaftliche Vorteil der Speicheranlagen liegt vor allem auch darin, dass praktisch nur Oberflächenabflüsse genutzt sowie umgelagert werden und Trinkwasserressourcen nur eine sehr untergeordnete Rolle spielen.

Eine besondere Rolle kommt auch der Weiterentwicklung von Anlagen zur Schneeerzeugung und insbesondere der laufenden Verbesserungen sowohl für eine Reduzierung des Energieverbrauches als auch zur Verbesserung der Schneequalität zu. Darauf wird in einem technischen Beitrag eingegangen und des Weiteren geht aus einer Statistik der Betreiber hervor, dass der Energieverbrauch je Kubikmeter erzeugtem technischen Schnee von ursprünglich 7 bis 3 kWh auf etwa 3 bis $1 \mathrm{kWh}$ reduziert werden konnte. In den letzten Jahren wurden sehr leistungsstarke Beschneiungsanlagen entwickelt, die eine Vollbeschneiung in nur wenigen Tagen ermöglichen.

Aus bautechnischer Sicht wird in dem Sonderheft über die Erfahrungen bei der Errichtung einer gröBeren Speicheranlage mit konventionellem Dichtungssystem und mit einer Asphaltbetondichtung berichtet sowie auf die Erfahrungen eines Betreibers von mehreren Speicheranlagen eingegangen.

Speicheranlagen bedürfen einer besonderen Betreuung und regelmäßigen Überwachung sowie Beobachtung zur Erfüllung der gestellten Sicherheitsanforderungen. Die für andere Wasserbauwerke übliche und allgemeine Philosophie in Österreich zur Stand- und Betriebssicherheit beruht auf dem „Drei-Säulen-Modell“ und betrachtet die Sicherheit von „Entwurf und Ausführung“, „Betrieb und Überwachung“ und „Vorkehrungen für Notfälle“. Nur durch eine sichere Betriebsführung und 
konsequente Überwachung von Speicheranlagen ist es möglich, Schäden und Versagensfälle mit Personen- und größerem Sachschaden zu verhindern. Um dies künftig zu gewährleisten und um den hohen Sicherheitsstandard in Österreich aufrecht halten zu können, kommt einer laufenden Ausbildung und Schulung des zuständigen Betriebspersonals sowie den Stauanlagen- oder Speicherverantwortlichen besondere Bedeutung zu. Durch die Vielzahl von bestehenden und neu geplanten Anlagen wurde unter Federführung der zuständigen Behörden im Jahre 2011 der neue „Leitfaden für das wasserrechtliche Behördenverfahren von Beschneiungsanlagen“ erstellt, in dem sowohl die Anforderungen an die Neuplanung samt erforderlichen Einreichunterlagen, sämtliche sicherheitsrelevanten Maßnahmen als auch die umfassenden Details für die laufenden und wiederkehrenden Überprüfungen festgelegt sind. Weiters wird auf die Wiederverleihung von Wasserrechten und den technischen Standard der bestehenden und neu zu genehmigenden Anlagen Bezug genommen.

Zu Genehmigungen von Beschneiungsanlagen und Speichern sind gegebenenfalls auch weitere Fachmaterien wie Umweltverträglichkeitsprüfungen, Naturschutzgesetze, Raumplanungsvorgaben, Alpenkonvention etc. zu berücksichtigen.

\section{Univ.-Prof. DI Dr. P. Tschernutter}

Technische Universität Wien

Institut für Wasserbau und Ingenieurhydrologie

Leiter Forschungsbereich Wasserbau und Hydraulisches Versuchslabor

Karlsplatz 13,1040 Wien

peter.tschernutter@kw.tuwien.ac.at 